\title{
Análisis y reflexión de prácticas evaluativas asociadas a la Resolución de Problemas en Matemáticas. Una perspectiva constructivista ${ }^{1}$
}

\author{
Analysis and reflection of assessment practices associated with \\ Problem Solving in Mathematics. A constructivist perspective \\ Análise e reflexão das práticas de avaliação associados à resolução \\ de problemas em Matemática. A perspectiva construtivista
}

Recibido: mayo de 2013

Aceptado: agosto de 2013

Mejía Osorio Jeny Alexandra²

\begin{abstract}
Resumen
La evaluación del aprendizaje ha de permitir un diálogo constructivo con los estudiantes, el calificar un resultado exclusivamente final pierde credibilidad sobre el proceso llevado a cabo en el aula dado que no ha de ser posible tener un acercamiento a los avances y dificultades de los estudiantes y por tanto no se analiza el proceso en su totalidad. En este sentido, desde la resolución de problemas se deben llevar a cabo procesos de construcción conceptual en diferentes momentos, que fortalezcan el trabajo cooperativo y donde se permita a los estudiantes estar en posibilidad de equivocarse, como medio para llevar a cabo un proceso dinamizador y reflexivo, a partir del cual se facilite la consecución de logros dentro de un proceso que busca la calidad. Y es a tal sentido, que apunta el presente estudio, en el cual mediante el trabajo colaborativo de estudiante-investigador y profesor se diseña una propuesta de evaluación para ser aplicada al desarrollo de un espacio de formación mediado por la resolución de problemas, teniendo en cuenta que se hace necesario reconocer un proceso en el que todos los entes que allí participan, son protagonistas del mismo.
\end{abstract}

Palabras clave: Practicas evaluativas; resolución de problemas; reconocimiento avances-dificultades; evaluación constructiva; trabajo colaborativo; enseñanza; planificación del profesor; evaluación.

\begin{abstract}
Learning assessment must enable constructive dialogue with students, branding outcome end only loses credibility on the process carried out in the classroom as it has not be possible to have an approach to the progress and difficulties of students and therefore not analyzed the whole process. In this sense, problem solving should be carried out conceptual building processes at different times, to strengthen cooperative work and where students are allowed to be in being wrong, as a means to conduct a dynamic process and reflective, from which to facilitate the accomplishment of achievement within a process that seeks quality. And it is this sense,
\end{abstract}


the present study aimed, in which through collaborative work and student-teacher researcher designing a proposed assessment to be applied to the development of a training mediated by problem solving, taking into note that it is necessary to recognize a process in which all entities that participate there are protagonists..

Keywords: Evaluative practices, resolution of problems, progress, difficulties recognition, evaluation constructive collaborative work, teaching, teacher planning, assessment.

\section{Resumo}

Avaliação da aprendizagem deve permitir um diálogo construtivo com os alunos, resultado final marca só perde credibilidade no processo realizado em sala de aula, uma vez que não é possível ter uma abordagem para o progresso e as dificuldades dos alunos e por conseguinte, não analisado todo o processo. Neste sentido, a resolução de problemas devem ser efectuados processos de construção conceptuais em momentos diferentes, para reforçar o trabalho cooperativo e onde os estudantes podem ser em ser errada, como um meio para realizar um processo dinâmico e reflexivo, a partir do qual a fim de facilitar a realização de realização dentro de um processo que busca a qualidade. E é neste sentido, o presente estudo teve como objetivo, em que através de um trabalho colaborativo e pesquisador aluno-professor projetar uma proposta de avaliação a ser aplicado para o desenvolvimento de uma formação mediada pela resolução de problemas, tendo em notar que é necessário reconhecer um processo em que todas as entidades que participam existem protagonistas.

Palavras-chave: Práticas avaliativas, a resolução de problemas, o progresso, o reconhecimento de dificuldades, a avaliação do trabalho colaborativo construtivo, ensino, planejamento de professores, avaliação.

\section{Presentación del problema}

Atendiendo a que al trabajar la resolución de problemas estamos considerando los procesos que allí se desarrollan como constructivistas y por lo mismo interesados en el aprendizaje objetivo de los estudiantes. Se reconoce que en pro de trabajar en el mejoramiento de las prácticas docentes, quedan algunos aspectos sobre los que desde lo planteado en el presente estudio se hace necesario profundizar. En tal sentido, se plantea la necesidad de cambiar la práctica discursiva en torno a la evaluación de los aprendizajes, al señalar las tensiones que en ésta se dan, reconociendo en estas que i) Con la evaluación y dada la normatividad, se busca evaluar el nivel de aprendizaje logrado por los estudiantes a través de prácticas evaluativas relacionadas con exámenes que arrojan un valor meramente numérico. ii) En el aula, se dan restringidos espacios para la socialización de avances e igualmente se hace un seguimiento reducido a diversos elementos usados para la comunicación (cuaderno del resolutor) iii) En ocasiones, no se evalúa el proceso en su totalidad, sino la finalización de éste. iv) Formas de evaluación como la autoevaluación, coevaluación (intragrupal e intergrupal) y heteroevaluación, no son tenidas en cuenta, con lo cual se restringe el fortalecimiento de un proceso reflexivo por parte de los estudiantes. v) Finalmente, en el trabajo desarrollado por los profesores en las aulas de clase, se evidencia aislamiento en el sentido que no se comparten experiencias y/o efectos de la puesta en práctica de diversidad de estrategias en pro de posibilitar mejores prácticas. Por lo que 
se hace necesario establecer espacios que permitan articular el trabajo y el compartir de experiencias.

De esta forma, vemos como desde la resolución de problemas, se deben llevar a cabo procesos de construcción conceptual en diferentes momentos y es el docente, en su papel de guía el responsable de buscar las estrategias adecuadas para cumplir tal fin. En tal sentido, podemos apreciar que el cambiar la evaluación implica cambios en las concepciones que tienen los profesores sobre la misma, mejorar las prácticas evaluativas no se reduce a realizar mejores pruebas sino más bien a revisar las propias prácticas con el fin de promover un cambio en las concepciones que sobre evaluación se tienen al respecto.

\section{Marco de referencia.}

Evaluación. Desde Giménez (2001), se hace posible tener una aproximación a lo que ha sido la transformación histórica de las prácticas evaluativas llevadas a cabo en el aula, al igual que a los modelos de evaluación que pueden ser implementados, mediante la puesta en práctica de un sistema multidimensional ${ }^{3}$. Es en Giménez, donde se reconoce que independientemente del modelo con el cual se trabaje, todo proceso de evaluación ha de tener el sentido de reconocer el nivel de logro alcanzado en el mismo. En tal sentido, se pone en escena un modelo integrador, orientativo-reflexivo que pone en funcionamiento una evaluación integrada en un proceso metodológico activo y participativo, en el que la función principal es orientar al estudiante y guiar su proceso, reflexionando sobre dicho desarrollo. Tal modelo, tiene dentro de sus elementos característicos centrarse en la globalidad del proceso de enseñanza-aprendizaje, reconocer el valor de dificultades, establecer un proceso continuado y basarse en un diseño constructivo del aprendizaje.

Resolución de problemas. En lo referente a la metodología de resolución de problemas, se hace uso de las etapas planteadas por Polya para dar solución a un problema, en lo referente a entendimiento del problema, diseño de un plan, ejecución de plan, visión retrospectiva y por otra parte, Mason, Burton \& Stacey (1982) dadas las fases en el proceso de resolución de problemas abordaje, ataque y revisión con sus respectivos rótulos ¿Qué es lo que sé?, atascado- ¡AJÁ!, Comprobar - Reflexionar, los cuales consigan los estudiantes en su cuaderno de resolutor previo desarrollo de un problema planteado.

Trabajo colaborativo. Se opta por tener en cuenta los saberes de profesor y estudiante investigador, dado que se tienen puntos de vista diferentes que permiten que al establecer una articulación entre lo que cada uno de éstos percibe, se logre emitir juicios sobre los razonamientos elaborados y la implementación de una evaluación crítica y reflexiva sobre los procesos que se llevan a cabo en el aula. Es por esto que, el trabajo colaborativo, "permite a los investigadores y profesores reflexionar sobre la acción de la enseñanza y aprender conjuntamente. Por ello posee un excelente potencial para cambiar las formas de trabajo de los que en ella participan" Devís (1996, citado por García \& Trigo 2000, p.8). Es así que la investigación colaborativa permite a investigadores y educadores investigar los problemas de la educación mediante el trabajo conjunto en la planificación, implementación y análisis de las tareas concernientes al problema planteado tal como lo es la evaluación.

\section{Metodología.}

Se plantea el diseño y puesta en práctica de una propuesta de evaluación que atiende a todos y cada uno de los requerimientos necesarios para propiciar un espacio mediado por la reflexión de la propia practica. Por consiguiente, se plantea la puesta en práctica de una propuesta de evaluación diseñada desde el trabajo colaborativo de investigador y profesor, y aplicada al espacio de formación de la LEBEM problemas de álgebra geométrica, el cual se fundamenta desde la resolución de problemas como metodología de enseñanza-aprendizaje. población y muestra. Grupo de estudiantes (3). 
Objeto matemático a evaluar. Diferentes formas de solución de los tres problemas griegos.

Perspectiva de investigación. Cualitativa, como medio para valorar el proceso de los estudiantes atendiendo a diversas habilidades reconocidas desde la comunicación.

Método de investigación. Investigación-acción dado que el implementar una intervención en la práctica profesional ha de reflejar sus resultados en una mejora.

Instrumentos. Revisión y/o puesta en práctica de una serie de instrumentos que buscan aportar evidencias al mejoramiento de las prácticas evaluativas que en el aula tienen lugar. Dentro de estos tenemos: syllabus del espacio de formación, grabaciones audiovisuales (clases, seminarios), protocolos de las sesiones de clase, entrevista semiestructurada (grupo investigado, profesor), producciones de estudiantes (cuaderno de resolutor, parciales, exposiciones).

\section{¿Cómo se estructura la Propuesta de evaluación?}

Con el fin de estructurar la utilización de cada uno de los elementos de la propuesta, y tomando como referente tanto los antecedentes consultados, como las características de la LEBEM en lo que a la metodología de resolución de problemas respecta, se plantea la implementación del modelo criterial expuesto por Giménez (1997), el cual como se ha mencionado previamente ha de ser crítico, orientador, integrador y multidimensional.

\section{Puesta en práctica del modelo.}

Dada la pregunta ¿Cómo poner en práctica el modelo? se incorporan determinados momentos a analizar en el desarrollo de la clase, tales como asesorías, socializaciones, cuaderno de resolutor y examen. En tal sentido, en el gráfico (Ver anexo 1) se exponen los componentes (crítico, orientador, integrador y multidimensional) centrales de la propuesta basada en el modelo criterial de Giménez (1997) "crítico, orientador, integrador, y multidimensional" (p.75), además de los elementos (perfil del aprendizaje, grados de dificultad, estructura unidad, entre otros) propios de cada uno de ellos, que dan evidencias a los componentes, como medio para analizar posteriormente su uso e implicaciones en el desarrollo de los momentos (cuaderno, asesorías, socializaciones y examen) previamente determinados, los cuales tienen como finalidad el reconocer y valorar el aprendizaje de los estudiantes, todo ello enmarcado dentro de una propuesta de evaluación continua

\section{Análisis de datos.}

Matrices descriptivas. Se hace uso de matrices descriptivas como un recurso útil para analizar los datos al relacionar dos tipos de categorías y analizar horizontal y verticalmente su desarrollo en el aula de clase, en lo referente al proceso de aprendizaje de los estudiantes.

Relación Elementos-Momentos-Componentes. En el gráfico (Ver anexo 1) se exponen los componentes centrales de la propuesta basada en el modelo criterial de Giménez (1997) "crítico, orientador, integrador y multidimensional” (p.75), además de los elementos propios de cada uno de ellos, que dan evidencias a los componentes, como medio para analizar su uso e implicaciones en el desarrollo de los momentos previamente determinados, los cuales tienen como finalidad el reconocer y valorar el aprendizaje de los estudiantes, todo ello enmarcado dentro de una propuesta de evaluación continua. De acuerdo con esto, en el Anexo 2 se observa la ruta que se siguió para analizar la información, atendiendo a que analizo independientemente cada uno de los componentes con sus respectivos efectos.

\section{Conclusiones.}

Coincidimos en que el objetivo de la evaluación se sitúa en concordancia con una postura holística, en la que el reconocimiento de un proceso que atienda a un sinfín de posibilidades, es la salida para la consecución de un espacio constructivo propio de la mejora y la innovación en el 
acto educativo Es así, que el modelo integrador, orientativo-reflexivo que se implementó, puso en funcionamiento una evaluación integrada a un proceso metodológico activo y participativo, en el que la función principal fue orientar al estudiante y guiar su proceso, reflexionando sobre dicho desarrollo. Desde la autorregulación, se reconoce la necesidad de implementar espacios para que se autorregule el propio aprendizaje, el sistema y el mismo profesor de forma tal que se posibilite el reflexionar sobre cada una de las acciones que allí dentro tienen lugar y sus implicaciones en el aprendizaje. En cuanto al papel del error en los procesos, se parte de la premisa de que se hace necesario el reconocerlos y potencializarlos con el fin de generar aprendizaje desde los mismos.

La puesta en práctica del trabajo colaborativo de estudiante-investigador y profesor se reconoce como una fuente eficaz de trabajo en conjunto atendiendo a que cada uno de los momentos destinados para el compartir de ideas y la búsqueda de soluciones a situaciones que se generaban en la clase, se convirtieron en escenarios propios de la reflexión y análisis, lo que desencadeno en una búsqueda de soluciones en conjunto, previa mirada desde diferentes perspectivas (estudiantes, profesor). De igual forma, los espacios propios de la reflexión docente, se constituyeron como escenarios en los que el dialogo fue el eje articulador al tener en cuenta saberes de profesor y estudiante investigador, valorando puntos de vista diferentes que permitieron establecer una articulación entre lo que cada uno de éstos percibía, posibilitando el emitir juicios sobre los razonamientos elaborados, en pro de una evaluación crítica y reflexiva sobre los procesos que se llevaron a cabo en el aula.es decir, tal trabajo, se tomó como un proceso interminable de mejora continua, en el que los resultados fueron utilizados en la solución de los problemas.

\section{Referencias}

García, J \& Trigo, E. (2000). Investigación colabo rativa $y$ formación de universitarios. Universidade da Coruña. Equipo de investigación "Kon-traste: creatividad y motricidad" Revista de Educación. No 323 (AÑO 2000)

Giménez (1997) Evaluación en Matemáticas. Una integración de perspectivas.

LEBEM (1999). Proyecto Curricular de Licenciatura en Educación Básica con Énfasis en Matemáticas. Documento General Acreditación Previa. Santafé de Bogotá, D.C., Septiembre de 1999.

Sanmartí, N. (1998). La evaluación de los aprendizajes. (Adaptado del capítulo 2 del libro: Gairin, J. \& Sanmartí, N. (1998) La evaluación institucional. Ministerio Educación. Argentina

Santos Trigo. L (1997) Principios y métodos de la resolución de problemas en el aprendizaje de las matemáticas. México D.F.: Iberoamérica. 


\section{Anexo 1}

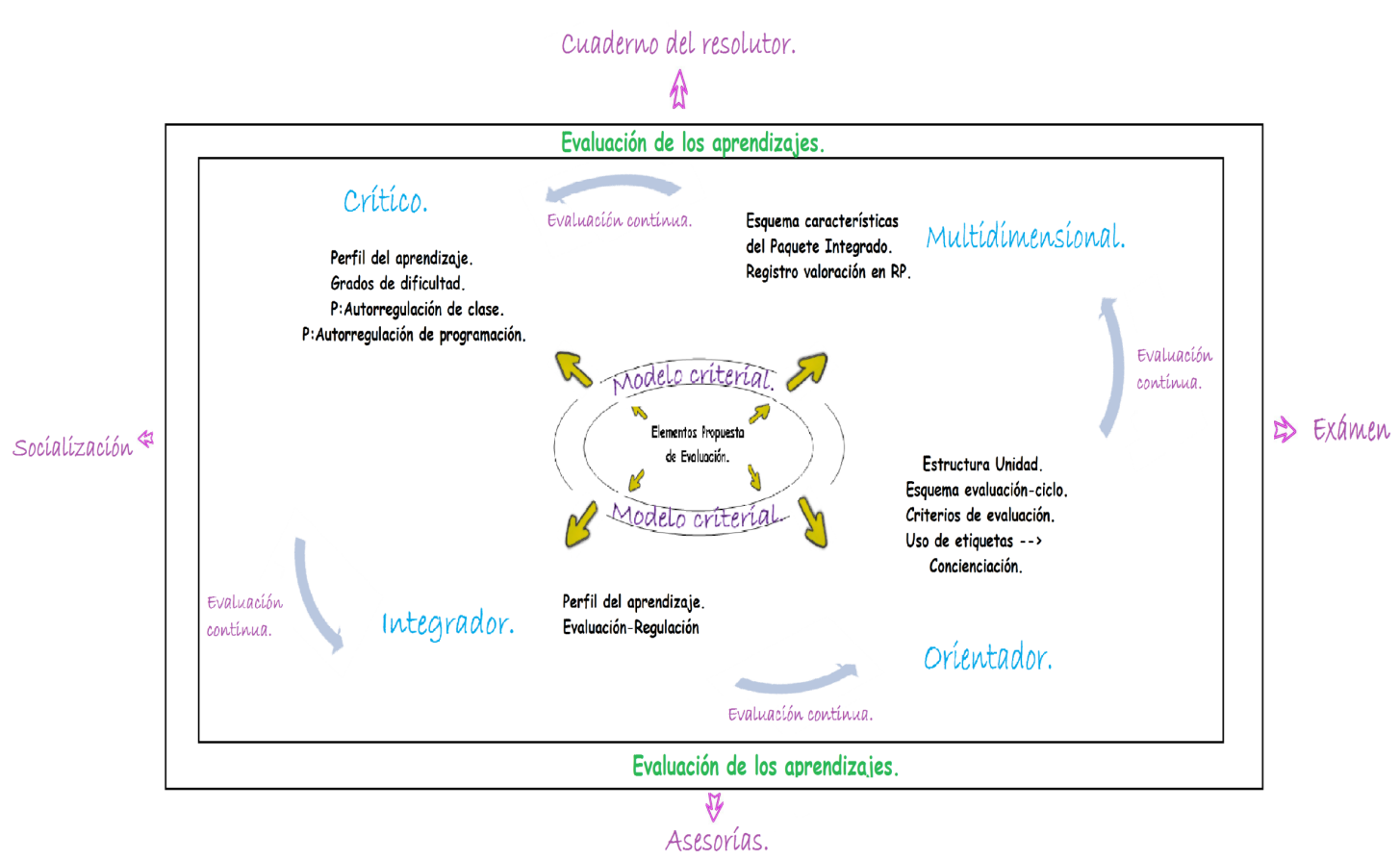

Fuente: Elaboración propia

Anexo 2

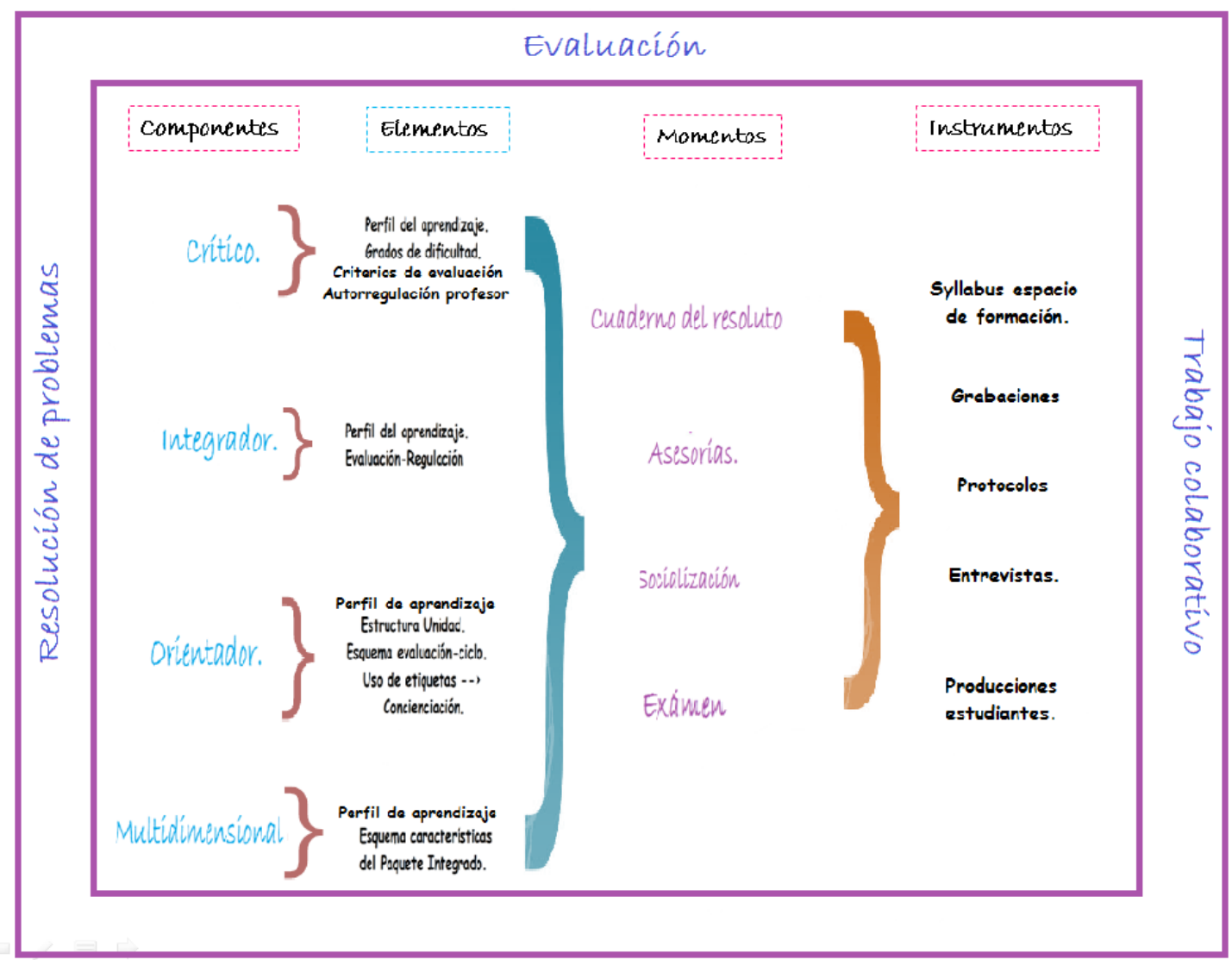

Fuente: Elaboración propia 